\title{
Aberrant expression of redox protein Ape1 in colon cancer stem cells
}

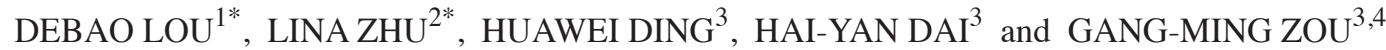 \\ ${ }^{1}$ Department of Pharmacy, Shanghai Eighth People's Hospital, Shanghai 200235; ${ }^{2}$ Department of Ophthalmology, \\ Renji Hospital, School of Medicine, Shanghai Jiao Tong University, Shanghai 200127; ${ }^{3}$ Shanghai Cancer Institute, \\ Shanghai Jiao Tong University, Shanghai 200240; ${ }^{4}$ Shanghai Institute for Pediatrics Research, \\ Xin Hua Hospital, Shanghai Jiao Tong University Shanghai of Medicine, Shanghai 200092, P.R. China
}

Received March 4, 2013; Accepted December 18, 2013

DOI: $10.3892 / \mathrm{ol} .2014 .1864$

\begin{abstract}
Ape1 is an important redox protein, essential for specific cytokine-induced signal transduction. Apel signaling is also important in regulating the growth of cancer cells, including colon cancer cells. The present study investigated whether Ape1 signaling plays a role in the regulation of colon cancer stem cell (CCSC) growth. The results showed that Ape1 was aberrantly expressed in CCSCs, as determined by quantitative (q) PCR assay. A laser confocal microscopy assay demonstrated that the Apel protein was mainly distributed in the nuclei, but not the cytoplasm, of the CSCs. Treatment of CCSCs with Apel redox inhibitor (E3330) significantly affected growth in vitro. In colon cancer xenograft mice, in vivo administration of E3330 enhanced tumor responses to the chemotherapeutic drug, 5-fluorouracil (5-FU). Furthermore, the combination of E3330 and 5-FU evidently increased the cytotoxicity of 5-FU in CSC growth. In the qPCR assay, the CCSCs were demonstrated to express the dominant ATP-binding cassette sub-family $G$ member 2 (ABC-G2), but not the multidrug resistance 1, genes. Thus, we hypothesized that drug resistance in CCSCs is mediated by ABC-G2. Since CSCs are involved in cancer metastasis, the Apel inhibitor may be a potential agent in the inhibition of colon cancer growth and metastasis.
\end{abstract}

\section{Introduction}

Ape1, also named Ref-1, is a multifunctional protein involved in apurinic/apyrimidinic (AP) endonuclease DNA base

Correspondence to: Professor Gang-Ming Zou, Shanghai Cancer Institute, Shanghai Jiao Tong University, 800 Dongchuan Road, Wenxuan Medicine Building, Room 442, Shanghai 200240, P.R. China

E-mail: gzou2003@yahoo.com

*Contributed equally

Key words: colon cancer, E3330, Ref-1, 5-fluorouracil, MDR1, ABC-G2 excision repair (BER) and redox activity. Apel is essential for specific cytokine-induced signal transduction, including that of interleukin-21 (1) and cluster of differentiation (CD)40L (2). CD40L triggers its receptor, CD40, which consequently mediates Apel nuclear translocation in human Burkitt's lymphoma cells (2). Apel is ubiquitously expressed at high basal levels. Previous studies in which Apel was disrupted using a gene targeting strategy have been used to determine its function in vivo (3). Embryos without Ape1 expression die in utero between implantation and day 6.5 , indicating normal embryonic development requires Apel (4). This gene is aberrant in various cancer cells (1). Our previous study demonstrated the aberrant expression of Ape1 in pancreatic cancer (5) and its role in tumor angiogenesis (6)

Redox balance underlies cellular homeostasis. Cancer initiation and progression have been associated with the disruption of redox balance and oxidative stress (7). Previously, Funato et al (8) demonstrated that reactive oxygen species can modulate signaling through the Wnt/ $\beta$-catenin pathway. The study highlighted new insights into the cross-talk between redox and $\mathrm{Wnt} / \beta$-catenin signaling in normal physiology and cancer. The Wnt signaling pathway may be regulated by redox signaling through redox-sensitive association of nucleoredoxin with dishevelled. A number of solid tumors contain substantial fractions of hypoxic cells that are relatively resistant to radiation therapy and certain cytotoxic drugs. Ape1, a nuclear protein, maintains the reduced state of Fos and Jun and promotes binding to activator protein 1 (AP-1). Nuclear extracts of HT29 colon cancer cells exposed to hypoxia show a markedly increased Ape1 protein content. Elevation of Ape1 mRNA levels occur as an early event following the induction of hypoxia and persists when cells are restored to a normally oxygenated environment (9). In addition, Ape1 Asp148Glu polymorphisms may be associated with the increasing risk of colorectal cancer (CRC) in a Turkish population (10).

Colon cancer is the second leading cause of cancer mortality in the United States, and $50 \%$ of patients with colon cancer develop synchronous or metachronous liver metastasis (11). Bleomycin (BLM) is used to treat various types of cancer, which generate cytotoxic double-strand breaks and abasic AP sites in DNA. The human Apel acts on abasic or 3'-blocking DNA lesions generated by ionizing radiation (IR) 
or BLM. It has been shown that cells are markedly sensitized to BLM cytotoxicity by partial Apel deficiency (30\% of normal levels), while resistance is largely restored by the expression of the unrelated yeast AP endonuclease, Apn1. It has also been demonstrated that apoptosis induced by BLM under Ape1 deficiency is partially p53-dependent in HCT116 colon cancer cells. Thus, the suppression or inhibition of Apel may be more efficacious as an adjuvant for BLM than for IR cancer therapy, particularly for tumors with a functional p53 pathway (12). These results pose the question of whether Apel expression is also important in the drug resistance of cancer stem cells (CSCs) in CRC. In the present study, Apel expression was examined in colon CSCs (CCSCs). It was found that CCSCs express dominant levels of the Ape1 gene and that the Ape1 inhibitor, E3330, enhances the chemotoxicity of 5-fluorouracil (5-FU) to these cells.

\section{Materials and methods}

Cell culture. The human colon cancer HT29 cell line was purchased from the American Type Culture Collection (Manassas, VA, USA). The cells were cultured in Dulbecco's modified Eagle's medium (DMEM) supplemented with $10 \%$ fetal bovine serum, $100 \mathrm{U} / \mathrm{ml}$ penicillin and $100 \mathrm{U} / \mathrm{ml}$ streptomycin (Invitrogen Life Technologies, Carlsbad, CA, USA).

Tumor sample collection. Fresh specimens of CRC tissue and adjacent non-cancerous tissues were obtained from patients who underwent surgical resection of preliminary CRCs and/or liver metastasis at the Department of Surgery, Shanghai Eighth People's Hospital (Shanghai, China). The study was approved by the Institutional Review Board of the hospital. A histological assessment was performed by pathologists using the World Health Organization standard grading system, with four categories (well-, moderately-, and poorly-differentiated and undifferentiated), was used (13).

Isolation of CCSCs. Fresh colorectal tumor specimens were immediately minced on ice, suspended in DMEM/F12 medium and dissociated with collagenase (both Invitrogen Life Technologies) and hyaluronidase (Calbiochem, La Jolla, CA, USA). Enzymatically disaggregated suspensions were filtered and washed three times with PBS and red blood cells were removed by Histopaque-1077 (Sigma-Aldrich, St. Louis, MO, USA). The resulting single tumor cells were placed under stem cell conditions in serum-free DMEM/F12 supplemented with human recombinant EGF and basic FGF (Invitrogen Life Technologies), and cultured on Ultra Low Attachment plates (Corning Inc., Corning, NY, USA). Single-cell suspensions in stem cell medium (1:10,000 cells/well) were analyzed for tumor sphere formation and numbers in each well were quantified after 14 days. Next, tumor spheres were disaggregated and reseeded to evaluate self-renewal by formation of secondary tumor spheres. Isolation of $\mathrm{CD} 133^{+} / \mathrm{ESA}^{+} \mathrm{CCSC}$ was performed by magnetic-activated cell sorting (MACS) or fluorescence-activated cell sorting (FACS).

Quantitative (q)PCR. Total RNA was extracted from the samples using TRIzol according to the manufacturer's instructions (Invitrogen Life Technologies). RNA was solubilized in
Table I. Primer sequences for qPCR.

\begin{tabular}{lc}
\hline Gene name & \multicolumn{1}{c}{ qPCR primers } \\
\hline Ape1 & \\
Forward & ACT TCA GGA GCT GCC TGG ACT \\
Reverse & AAT GCA GGT AAC AGA GAG TGG GA
\end{tabular}

qPCR, quantitative PCR.

$\mathrm{ddH}_{2} \mathrm{O}$, and the nucleic acid concentration was measured by a Nano Drop 2000 (Thermo Fisher Scientific, Waltham, MA, USA). cDNA was prepared using the PrimeScript kit (Takara Biotechnology (Dalian) Co., Ltd., Dalian, China). In each case, random and oligo(dT) primers were used. qPCR analysis was performed with an ABI 7300 (Applied Biosystems, Inc., Foster City, CA, USA), using SYBR Premix EX Taq as the reaction reagent. The relative quantities of the genes were calculated with $\beta$-actin as a reference, using the following formula: Relative quantity of gene $=2^{[-(\mathrm{CtGene}-\mathrm{Ct} \beta \text {-actin) }}$. Primer sequences are listed in Table I.

Laser confocal microscopy. For experiments on Ape1-Flag localization, a sequential protocol of immunofluorescence was used, based on labeled goat anti-mouse isotype-specific secondary antibodies. The cells were fixed in 4\% (wt/vol) paraformaldehyde for $20 \mathrm{~min}$ at room temperature, permeabilized for 5 min with PBS-0.25\% (wt/vol) Triton X-100 and incubated for $30 \mathrm{~min}$ with $5 \%$ normal goat serum in PBS-0.1\% (wt/vol) Triton X-100 (blocking solution) to block the non-specific binding of the antibodies. The cells were then incubated with the mouse monoclonal anti-FLAG (IgG1; 1:1,000; Sigma-Aldrich) or -APE1 (IgG2b; 1:30; Cell Signalling Technology, Danvers, MA, USA) antibodies, in blocking solution, for 2.5 h. Following washing, the cells were incubated for 90 min with Alexa Fluor 488-conjugated goat anti-mouse, IgG1 or Alexa Fluor 546-conjugated goat anti-mouse, IgG2b (1:200; Invitrogen Life Technologies) secondary antibodies. The preparations were then washed with PBS three times for $5 \mathrm{~min}$ each in the dark. Nuclei were stained by $5 \mathrm{~min}$ of incubation in a 300-nM solution of 4',6-diamidino-2-phenylindole dihydrochloride (Sigma-Aldrich) in PBS. Next, the preparations were washed three times in PBS for $5 \mathrm{~min}$. The microscope slides were mounted onto slides in Mowiol 4-88 (Sigma-Aldrich), supplemented with DABCO (1:5; SigmaAldrich), as an antifade reagent. Coverslips were visualized by a Leica TCS SP laser scanning confocal microscope (Leica, Mannheim, Germany).

Cell growth assay of CCSC in vitro in response to 5-FU and E3330. CCSCs were cultivated with a cell density of $1 \times 10^{4}$ cells per well in a 96-cell plate overnight. On the second day, the cells were treated with E3330 alone (concentration of $1 \mu \mathrm{M}$ ) or with the addition of various doses of 5-FU (1 or $5 \mu \mathrm{g} / \mathrm{ml}$ ) for $48 \mathrm{~h}$. Next, MTT assays were performed to analyze cell viability.

Animal and tumor models. Female, four-week-old $\left(\mathrm{mu}^{+} / \mathrm{nu}^{+}\right)$ mice were obtained from the Animal Center of Chinese 
Academy of Science (CAS, Shanghai, China) and maintained under the Institutional guideline of CAS. The CSCs that formed spheres were dissociated by collagenase and washed with PBS twice. The dissociated sphere cells $\left(5 \times 10^{5}\right)$ were injected subcutaneously with Matrigel (BD Biosciences, Franklin Lakes, NJ, USA) in a total volume of $100 \mu$ l. Tumor sizes were calculated once a week for 20 weeks according to the following formula: Tumor size $=\pi / 6 \mathrm{x}$ larger diameter $\mathrm{x}$ smaller diameter. Chemotherapy sensitization was assessed, and well-established tumors were generated by subcutaneous injection of sphere cells. Six mice per group were treated intraperitoneally with $5-\mathrm{FU}(15 \mathrm{mg} / \mathrm{kg} / \mathrm{day}, 5$ days a week for 2 weeks) alone or in combination with intratumoral injections of E3330 (1.5 mg/kg/day, 5 days a week for 2 weeks).

Statistical analysis. Statistical analysis was performed using the SPSS 9.0 statistical software package (SPSS, Inc., Chicago, IL, USA), and Student's t-test was used to test the probability of significant differences between samples. $\mathrm{P}<0.05$ was considered to indicate a statistically significant difference.

\section{Results}

Apel is expressed in CCSCs. To elucidate the role of Ape1 redox signaling in CSC regulation, the expression level of Ape1 was first examined in CCSCs. CD133 $/ \mathrm{ESA}^{+}$CCSCs were isolated from bulk cancer cells by FACS or MACS. The expression of the Ape1 gene in the CCSCs was examined by qPCR assay in the cells isolated from three independent patients. As shown in Fig. 1, all cells were found to express higher levels of the Ape1 gene compared with $\mathrm{CD} 133 \% \mathrm{ESA}^{+}$non-stem cells or adjacent non-cancerous tissues (Fig. 1A). Ape1 protein expression was further examined in these CSCs using laser confocal microscopy. The expression of Ape1 protein in the CCSCs was confirmed by laser confocal microscopy, and was mainly distributed within the nuclei of the CCSCs (Fig. 1B).

Inhibition of Apel redox function by E3330 affects CCSC growth. E3330 is a specific inhibitor of the Ape1 redox domain (3). Our previous study described the role of E3330 in inhibiting the growth of digestive tract cancer cells, such as pancreatic cancer cells (4). In the present study, the role of E3330 in the regulation of CSC growth was examined. The treatment of CCSCs with E3330 was found to significantly reduce the growth of cells in cultures treated with an E3330 concentration of $10 \mu \mathrm{M}$ (Fig. 2A). Moreover, the addition of a non-cytotoxic (i.e. cell growth-inhibiting) dose of E3330 $(1 \mu \mathrm{M})$ was found to significantly enhance the chemotoxicity of 5-FU in CCSCs in vitro (Fig. 2B).

Combination of E3330 with 5-FU enhances the cytotoxicity of 5-FU in colon cancer chemotherapy. To test the hypothesis that the combination of E3330 with 5-FU enhances the cytotoxicity of 5-FU in colon cancer chemotherapy in vivo, E3330 was analyzed to investigate whether it enhances the chemotherapuetic efficiency in CCSC xenograft models. 5-FU is a standard drug in the chemotherapy of colon cancer. Tumor xenografts generated by sphere cell injection were exposed to intraperitoneal administration of 5-FU plus intratumoral injections of ethanol or E3330. The tumor response to chemotherapeutic drugs was enhanced by E3330, whereas ethanol, as a control pretreatment, did not prevent tumor outgrowth. Specifically, the combined administration of E3330 and 5-FU resulted in a marked antitumor effect, whereas 5-FU alone did not (Fig. 3).

Multidrug resistance 1 (MDRI) and ATP-binding cassette sub-family $G$ member 2 (ABC-G2) gene expression in $C D 133^{+} / E S A^{+}$CCSCs. The overexpression of human Ape1, a key enzyme in the DNA BER pathway, is often associated with tumor cell resistance to various anticancer drugs. It has been previously indicated that Apel stably interacts with the Y-box-binding protein 1 (YB-1) and acts as its coactivator for the expression of the multidrug resistance gene, MDR1, thereby causing drug resistance. Ape1 is also stably associated with the basic transcription factor, RNA polymerase II, and the coactivator, $\mathrm{p} 300$, on the endogenous MDR1 promoter (11). MDR1 gene expression was examined by qPCR in $\mathrm{CD}_{133} / \mathrm{ESA}^{+} \mathrm{CCSC}$. Compared with $\mathrm{CD} 133 \% \mathrm{ESA}^{+}$colon cancer cells of tumor tissue, $\mathrm{CD} 133^{+} / \mathrm{ESA}^{+} \mathrm{CCSC}$ were found to express extremely low levels of the MDR1 gene. Therefore, other drug resistance genes were examined and the ABC-G2 gene was found to be expressed dominantly in $\mathrm{CD}_{133^{+}} / \mathrm{ESA}^{+}$ CCSCs, but not in $\mathrm{CD}^{2} 33^{-} / \mathrm{ESA}^{+}$cancer cells derived from cancer tissue (Fig. 4).

\section{Discussion}

Apel is a multifunctional enzyme involved in DNA BER and protein redox regulation. This redox protein is overexpressed in various types of tumor tissues and cells, including lung (15) and bladder (16) cancer cells. However, the mechanism of expression of this redox protein in CSCs currently remains unclear. In the present study, the aberrant expression of Ape1 was identified in CCSCs by the examination of primary human colon cancer tissues. Previously, CSCs have been shown to be associated with drug resistance and the failure of chemotherapy in cancer, but the mechanism in this regard has not been fully demonstrated (17). Overexpression of the Ape1 gene in pathological stem cells is associated with drug resistance. It has been previously demonstrated that drug-induced Ape1 acetylation, which is mediated by $\mathrm{p} 300$, enhances the formation of the acetylated Ape1/YB-1/p300 complex on the MDR1 promoter. Enhanced recruitment of this complex increases MDR1 promoter-dependent luciferase activity and its endogenous expression in HEK 293T cells (14). However, the present study showed that Apel may not regulate drug resistance through MDR1, but alternatively through ABC-G2 in CSCs.

The ABC-G2 gene is also highly expressed in hematopoietic stem cells, but this gene is turned off in the majority of committed progenitor and mature blood cells (18). CSCs possess high levels of $A B C$ transporters. MDR1, which is also named $\mathrm{ABC}-\mathrm{B} 1$, is a transmembrane transporter system, which actively pumps cytotoxic drugs out of the cell. Although MDR1 acquired in vitro is different from MDR1 acquired in vivo, it has important consequences on the metastatic behavior and cellular phenotype. The MDR1-negative human colonic cancer HT29 cell line is more malignant than its MDR1-overexpressing variant (HT29 MDR1-positive). When implanted subcutaneously into SCID mice, undifferentiated 
A

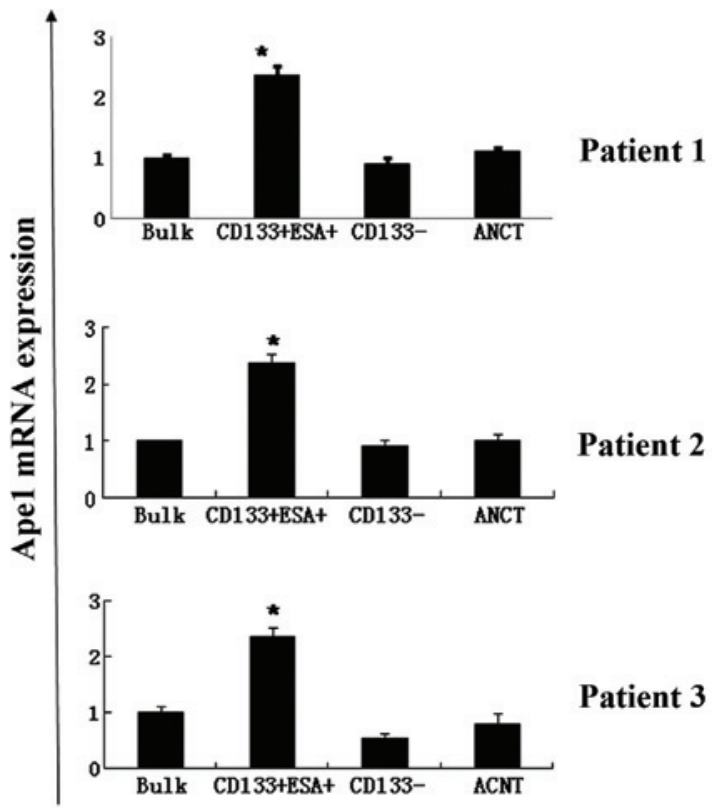

B

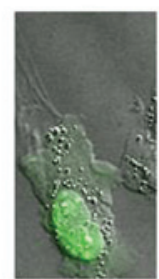

Figure 1. Expression of Ape1 gene in $\mathrm{CD} 133^{+} / \mathrm{ESA}^{+} \mathrm{CCSC}$ examined by quantitative (q)PCR and laser confocal microscopy. (A) $\mathrm{CD}^{2} 33^{+} / \mathrm{ESA}^{+}$ CCSCs were isolated from fresh colon cancer tissue by FACS. The total RNA was extracted and qPCR was performed. (B) Apel gene expression (green) in CCSCs examined by laser confocal microscopy. ${ }^{*} \mathrm{P}<0.05$, vs. control cells. CCSCs, colon cancer stem cells; FACS, fluorescence-activated cell sorting.

A

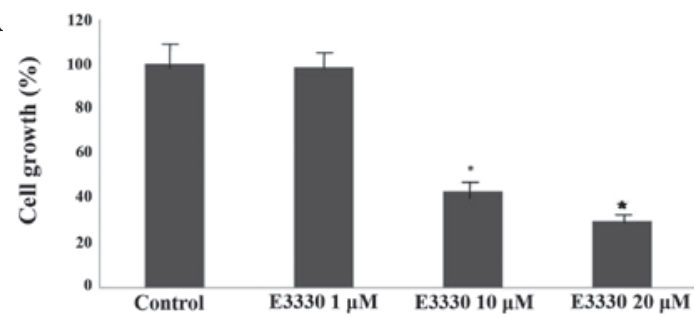

B

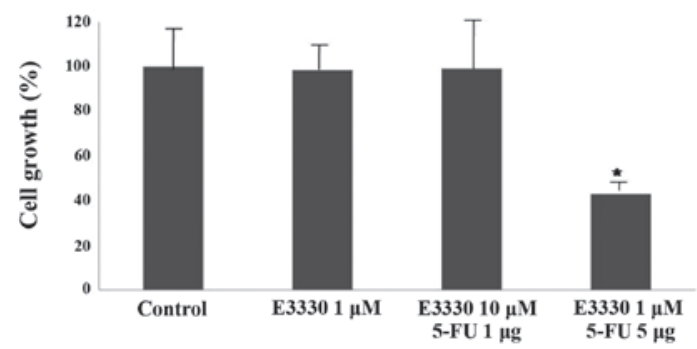

Figure 2. Effect of Ape1 redox inhibitor on $\mathrm{CD}_{133} / \mathrm{ESA}^{+} \mathrm{CCSC}$ growth (A) Results showing dose-dependent inhibition of CCSC growth by E3330 $\mathrm{CD}_{133}{ }^{+} / \mathrm{ESA}^{+} \mathrm{CCSC}$ were treated with various doses of $\mathrm{E} 3330$ for $72 \mathrm{~h}$. MTT assays were then performed to analyze cell viability. (B) E3330 enhanced the cytotoxic effect of 5-FU in suppressing CCSC growth in vitro. E3330 at a non-cytotoxic dose $(1 \mu \mathrm{M})$ was applied in this experiment. Data are presented as the mean $\pm \mathrm{SD}$ of three independent experiments. ${ }^{*} \mathrm{P}<0.05$ vs. control. CCSCs, colon cancer stem cell; 5-FU, 5-fluorouracil.
5FU: $15 \mathrm{mg} / \mathrm{kg} /$ day 5 days per week, $x 2$ weeks E3330: $1.5 \mathrm{mg} / \mathrm{kg} / \mathrm{day}, 5$ days per week, $\times 2$ weeks

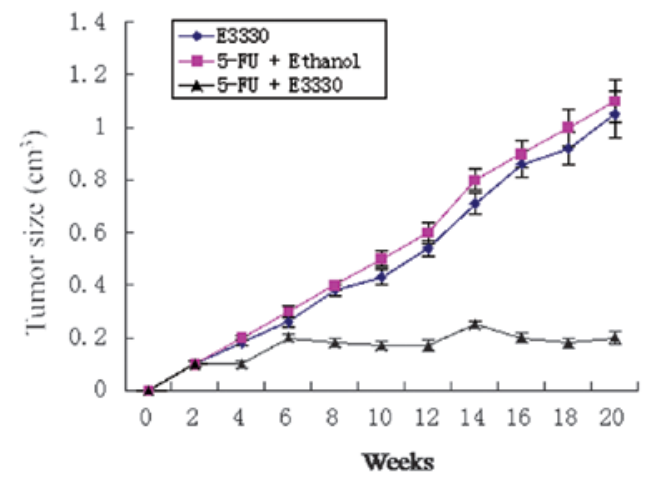

Figure 3. Combinated effect of E3330 and 5-FU in the suppression of $\mathrm{CD}_{133}{ }^{+} / \mathrm{ESA}^{+} \mathrm{CCSC}$ growth in vivo. Tumor size following intratumoral injection of ethanol alone (vehicle control), 5-FU or 5-FU/E3330 post-treatment. Data are presented as mean tumor size \pm SD of four independent sets of five tumors per group. Each set of tumors was exhibited using cells from different tumors. 5-FU was administered at $15 \mathrm{mg} / \mathrm{kg} /$ day, 5 days per week for 2 weeks; and E3330 administered at $1.5 \mathrm{mg} / \mathrm{kg} /$ day, 5 days per week for 2 weeks. 5-FU, 5-fluorouracil; CCSC, colon cancer stem cell.

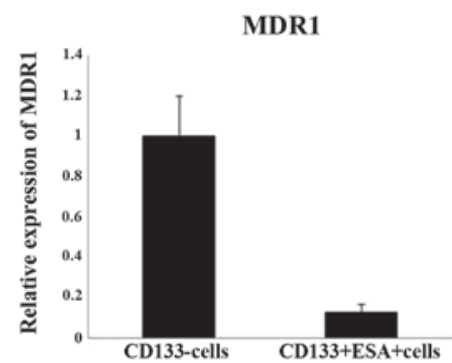

ABC-G2

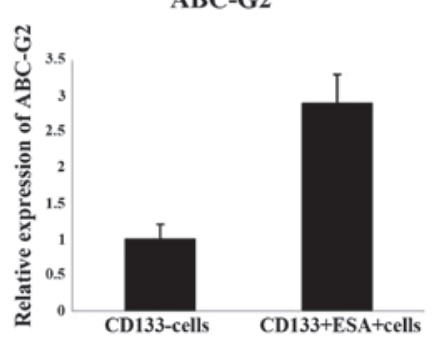

Figure 4. Drug resistance gene expression in CCSCs. MDR1 and ABC-G2 gene expression was examined by quantitative (q)PCR in $\mathrm{CD} 133^{+} / \mathrm{ESA}^{+}$ CCSCs isolated from fresh CRC tissue. Data are presented as the mean $\pm \mathrm{SD}$ of values for three patients. ${ }^{*} \mathrm{P}<0.05$, vs. CD133 cells. CCSC, colon cancer stem cell; CRC, colorectal cancer; MDR1, multidrug resistance 1; ABC-G2, ATP-binding cassette sub-family G member 2 .

signet ring carcinomas are produced by HT29 MDR1-negative cells, while tumors with tubular structures, but without signet ring cells, are formed by HT29 MDR1-positive cells (MDR1 overexpression in HT29 colon cancer cells grown in SCID mice) (19). The results of the current study are consistent with this hypothesis, and CCSCs were found to express extremely low levels of the MDR1 gene, but high levels of ABC-G2. Thus, we hypothesized that ABC-G2, but not MDR1, is key in the drug resistance of CSCs.

$5-\mathrm{FU}$ is one of the major drugs in chemotherapy for CRC, but CCSCs are resistant to 5-FU (20). CCSCs may be 
expanded as tumor spheres in vitro using a serum-free medium containing EGF and bFGF. Such tumor spheres contain CSCs, cancer progenitors and early precursors, presenting the best-characterized method to expand an enriched population of tumorigenic cells (21). Wnt activity is high in CD133-positive DLD1 colon cancer cells compared with CD133-negative DLD1 colon cancer cells. The Wnt activity of CD133-positive colon cancer cells may be upregulated by 5-FU, while blocking this activity may reverse the drug sensitivity of these cells to 5-FU (22). Therefore, the combination of Ape1 and Wnt inhibitors exhibits an improved effect in inhibiting colon cancer cell proliferation. This indicates that the combination of these inhibitors may improve the efficacy of future CRC therapy. We aim to analyze the association between Apel and Wnt signaling in CCSCs in future studies.

In summary, the present study identified the aberrant expression of Ape1 in CCSCs, and the inhibition of its redox activity is likely to enhance the cytotoxicity of 5-FU against these cells.

\section{Acknowledgements}

The present study was partly supported by research grants from the Shanghai Cancer Institute and Shanghai Natural Science Foundation (no. 11ZR1434800) provided by the Shanghai Science and Technology Commission. The authors would like to thank Dr De-Jian Dai from the Department of Surgery in Shanghai Eighth People's Hospital who provided the colon cancer tumor samples for the present study, and Dr Zhihua Chen for technical assistance with the processing of the cancer tissue.

\section{References}

1. Juliana FM, Nara H, Onoda T, Rahman M, Araki A, Jin L, et al: Apurinic/apyrimidinic endonuclease1/redox factor-1 (Ape1/Ref-1) is essential for IL-21-induced signal transduction through ERK1/2 pathway. Biochem Biophys Res Commun 420: 628-634, 2012.

2. Merluzzi S, Gri G, Gattei V, Pagano M and Pucillo C: APE/Ref-1 makes fine-tuning of CD40-induced B cell proliferation. Mol Immunol 45: 3731-3739, 2008.

3. Xanthoudakis S, Smeyne RJ, Wallace JD and Curran T: The redox/DNA repair protein, Ref-1, is essential for early embryonic development in mice. Proc Natl Acad Sci USA 93: 8919-8923, 1996.

4. Zou GM, Luo MH, Reed A, Kelley MR and Yoder MC: Ape1 regulates hematopoietic differentiation of embryonic stem cells through its redox functional domain. Blood 109: 1917-1922, 2007.

5. Zou GM and Maitra A: Small-molecule inhibitor of the AP endonuclease 1/REF-1 E3330 inhibits pancreatic cancer cell growth and migration. Mol Cancer Ther 7: 2012-2021, 2008.
6. Zou GM, Karikari C, Kabe Y,Handa H, Anders RA and Maitra A: The Ape-1/Ref-1 redox antagonist E3330 inhibits the growth of tumor endothelium and endothelial progenitor cells: therapeutic implications in tumor angiogenesis. J Cell Physiol 219: 209-218, 2009.

7. Grek CL and Tew KD: Redox metabolism and malignancy. Curr Opin Pharmacol 10: 362-368, 2010.

8. Funato $\mathrm{Y}$, Michiue T, Asashima $\mathrm{M}$ and Miki $\mathrm{H}$ : The thioredoxin-related redox-regulating protein nucleoredoxin inhibits Wnt-beta-catenin signalling through dishevelled. Nat Cell Biol 8: 501-508, 2006.

9. Yao KS, Clayton M and O'Dwyer PJ: Interaction of heat and hypoxia in modulating transcription of DT diaphorase in human colon adenocarcinoma cells. Cell Growth Differ 5: 125-131, 1994.

10. Canbay E, Cakmakoglu B, Zeybek U, et al: Association of APE1 and hOGG1 polymorphisms with colorectal cancer risk in a Turkish population. Curr Med Res Opin 27: 1295-1302, 2011.

11. Jemal A, Siegel R, Ward E, Hao Y, Xu J, Murray T and Thun MJ: Cancer statistics, 2008. CA Cancer J Clin 58: 71-96, 2008.

12. Fung H and Demple B: Distinct roles of Apel protein in the repair of DNA damage induced by ionizing radiation or bleomycin. J Biol Chem 286: 4968-4977, 2011.

13. Denk H and Klimpfinger M: Colorectal cancer: classification and aspects of the proliferation kinetics. Wien Med Wochenschr 138: 288-292, 1988 (In German).

14. Sengupta S, Mantha AK, Mitra S and Bhakat KK: Human AP endonuclease (APE1/Ref-1) and its acetylation regulate YB-1-p300 recruitment and RNA polymerase II loading in the drug-induced activation of multidrug resistance gene MDR1. Oncogene 30: 482-493, 2011.

15. Puglisi F, Aprile G, Minisini AM, Barbone F, Cataldi P, Tell G, et al: Prognostic significance of Ape1/ref-1 subcellular localization in non-small cell lung carcinomas. Anticancer Res 21: 4041-4049, 2001

16. Sak SC, Harnden P, Johnston CF, Paul AB and Kiltie AE: APE1 and XRCC1 protein expression levels predict cancer-specific survival following radical radiotherapy in bladder cancer. Clin Cancer Res 11: 6205-6211, 2005.

17. Dean M, Fojo T and Bates S: Tumour stem cells and drug resistance. Nat Rev Cancer 5: 275-284, 2005.

18. Scharenberg CW, Harkey MA and Torok-Storb B: The ABCG2 transporter is an efficient Hoechst 33342 efflux pump and is preferentially expressed by immature human hematopoietic progenitors. Blood 99: 507-512, 2002.

19. Schumacher U, Nehmann N, Adam E, Mukthar D, Slotki IN, Horny HP, et al: MDR-1-overexpression in HT 29 colon cancer cells grown in SCID mice. Acta Histochem 114: 594-602, 2012.

20. Pang R, Law WL, Chu AC, Poon JT, Lam CS, Chow AK, et al: A subpopulation of CD26+ cancer stem cells with metastatic capacity in human colorectal cancer. Cell Stem Cell 6: 603-615, 2010.

21. Lombardo Y, Scopelliti A, Cammareri P, Todaro M, Iovino F, Ricci-Vitiani L, et al: Bone morphogenetic protein 4 induces differentiation of colorectal cancer stem cells and increases their response to chemotherapy in mice. Gastroenterology 140: 297-309, 2011.

22. Deng Y, Su Q, Mo J, Fu X, Zhang Y and Lin EH: Celecoxib downregulates CD133 expression through inhibition of the Wnt signaling pathway in colon cancer cells. Cancer Invest 31: 97-102, 2013 